\title{
Corrective surgery for kyphosis in a case of Gaucher's disease without history of vertebral compression fractures
}

\author{
Kenyu Ito ${ }^{122}$, Noriaki Kawakami ${ }^{1)}$, Taichi Tsuji ${ }^{1)}$, Tetsuya Ohara ${ }^{1)}$, Toshiki Saito ${ }^{1)}$, Ryoji Tauchi ${ }^{1)}$ and Kazuaki Morishita ${ }^{1)}$ \\ 1) Department of Orthopaedic Surgery and Spine Center, Meijo Hospital, Nagoya, Aichi, Japan \\ 2) Department of Orthopaedic Surgery, Nagoya University Graduate School of Medicine, Nagoya, Aichi, Japan
}

\begin{abstract}
:
Introduction: Gaucher's disease is a congenital metabolic disorder characterized by the accumulation of glucocerebroside in the reticuloendothelial system. Its clinical manifestations include splenomegaly, osteopenia, and pathological fractures. Cases of patients with kyphotic deformities caused by pathological vertebral compression fractures associated with Gaucher's disease are well reported. However, there has been no report regarding surgical treatment of kyphotic deformity caused by Gaucher's disease without compression fractures. In the present report, we describe surgical treatment for kyphotic deformity caused by Gaucher's disease without a past history of vertebral compression fractures.

Case Report: The patient was diagnosed with Gaucher's disease at the age of 15 months. The patient was a 10-year-old girl with progressive kyphosis $\left(84^{\circ}\right.$ between T6 and L3, with T12 as the apical vertebra) without compression fractures. Although the patient had been treated using a brace since the age of 3 years, the kyphosis progressed to the point where corrective surgery was required. We initially performed T3-L3 posterior spinal fusion, followed by anterior fusion 3 months later, which corrected the kyphosis to $35^{\circ}$. Postoperatively, the patient suffered fractures of the upper and lower extremities but did not have spinal fractures.

Conclusions: Two-stage anterior/posterior combined correction and fusion was performed in a patient with kyphotic deformity caused by Gaucher's disease without compression fractures. Because of bone fragility in Gaucher's disease, careful selection of the fusion range and postoperative therapy was necessary.

Keywords:

Gaucher's disease, kyphosis, compression fracture, posterior fusion, anterior fusion, metabolic disorder, fusion

Spine Surg Relat Res 2017; 1(4): 222-224

dx.doi.org/10.22603/ssrr.1.2017-0038
\end{abstract}

\section{Introduction}

Gaucher's disease is a congenital metabolic disorder characterized by genetically decreased activity of glucocerebrosidase whereby glucocerebroside accumulates in the reticuloendothelial system (the liver, spleen, bone marrow, etc.). It is an autosomal recessive hereditary disease. The prevalence rate is approximately $1 / 57,000$ births $^{2}$. The predominant clinical manifestations of Gaucher's disease include splenomegaly, osteopenia, and pathological fractures.

Cases of patients with kyphotic deformities because of pathological vertebral compression fractures associated with Gaucher's disease are well reported. To our knowledge, there has been no report regarding surgical treatment of kyphotic deformity caused by Gaucher's disease without compression fractures ${ }^{3-5)}$.

\section{Case Report}

History: At the age of 15 months, the patient developed hepatosplenomegaly and was diagnosed with Gaucher's disease, subsequently receiving enzyme-replacement therapy. Kyphosis was identified at the age of 3 years, and the patient began brace treatment, however the kyphosis progressed.

Patient description: The patient was a 10-year-old female, $129-\mathrm{cm}$ tall, weighing $30 \mathrm{~kg}$, and menarche had occurred three months earlier (Fig. 1). She exhibited mild weakness of the lower extremities and was able to walk with support. X-ray imaging revealed a Risser sign 1, kyphosis of $84^{\circ}$ be-

Corresponding author: Noriaki Kawakami, nupriver@sea.plala.or.jp

Received: May 13, 2017, Accepted: July 15, 2017

Copyright (C) 2017 The Japanese Society for Spine Surgery and Related Research 

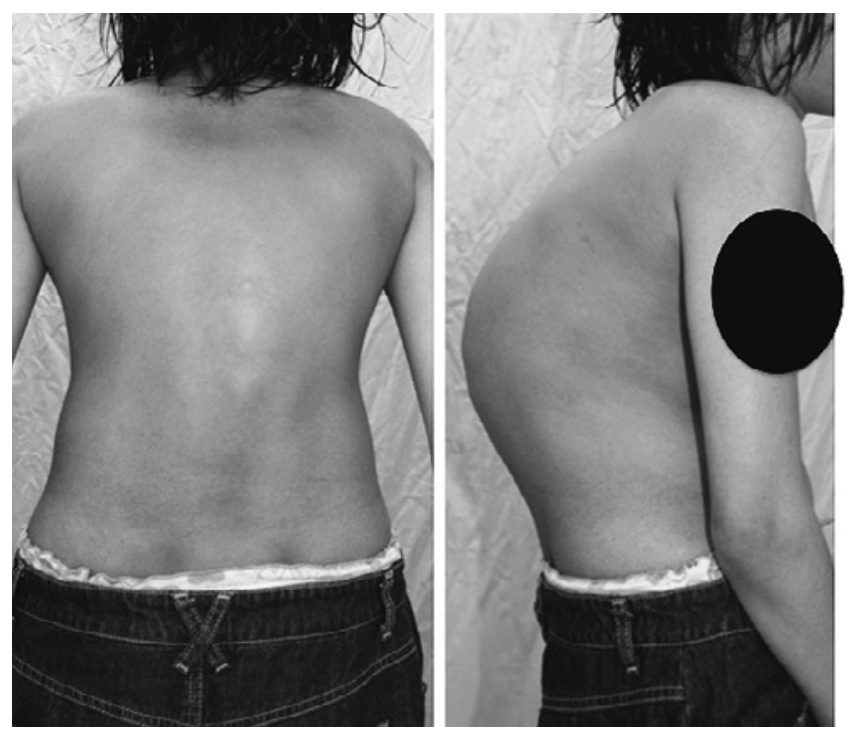

Figure 1. Patient's appearance. Ten-year-old female with a humped back.
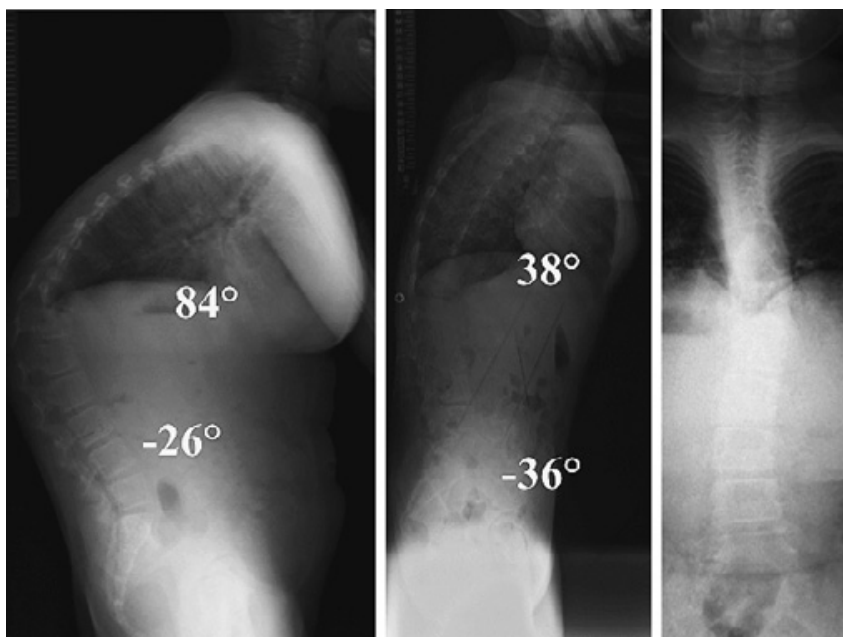

Figure 2. Preoperative radiograph. X-ray imaging showed kyphosis of $84^{\circ}$ between T6 and L3 with T12 as the apical vertebra and kyphosis was corrected to $38^{\circ}$ under traction. Local kyphosis was $51^{\circ}$ between $\mathrm{T} 11$ and $\mathrm{L} 1$ with slight scoliosis in anteroposterior view.

tween T6 and L3, with T12 as the apical vertebra, and kyphosis was corrected to $38^{\circ}$ under manual traction. Local kyphosis was $51^{\circ}$ between $\mathrm{T} 11$ and L1 with slight scoliosis in the anteroposterior view (Fig. 2). Computed tomography and magnetic resonance imaging revealed anterior vertebral wedging between T11 and L1 without any apparent compression fractures (Fig. 3a, b).

Surgery: Two-stage surgery was planned. First, T3-L3 posterior correction with T12 pedicle subtraction osteotomy was performed. We reinforced all the laminae using wires. The surgical time was $340 \mathrm{~min}$, and the estimated blood loss was $1,198 \mathrm{ml}$. The patient developed pneumonia on the second day after surgery, but her symptoms improved within 5 days.

Three months after the posterior fusion procedure, ante-

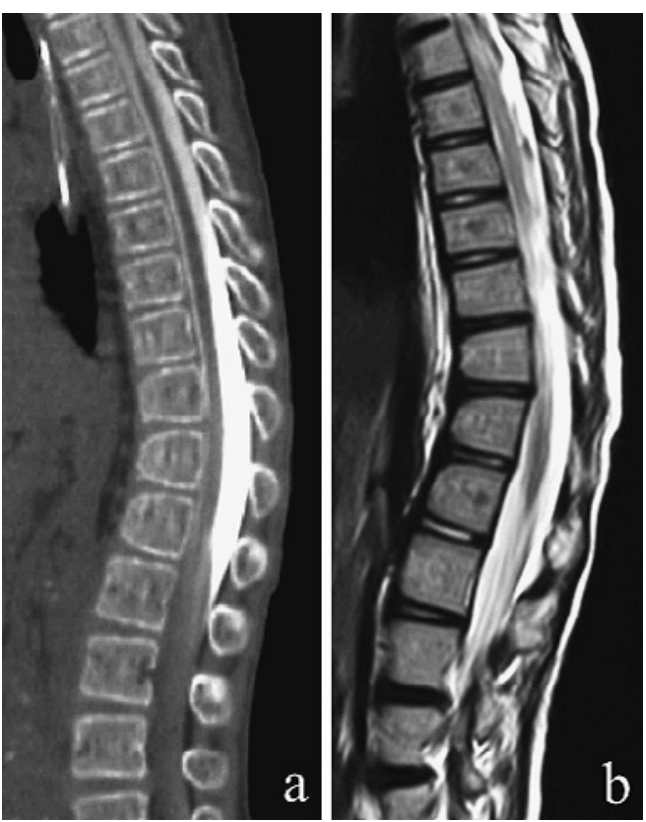

Figure 3. (a) Computed tomography myelogram. (b) Magnetic resonance imaging. Anterior vertebral wedging was recognized between T11 and L1, with T12 as its center but without any apparent vertebral compression fractures. The patient did not have spinal canal stenosis eitherr.

rior fusion was performed. T12 vertebra, L1-L2, and L2-L3 intervertebral discs were removed, and bone grafts were placed. The surgical time was $162 \mathrm{~min}$, with an estimated blood loss of $218 \mathrm{ml}$. The patient's physical status enabled immediate extubation after the surgery. She was discharged 14 days after the surgery.

Postoperative course: Six months after the surgery, the patient suffered right femoral, left big toe, right 3rd toe, right big toe, and left ankle malleolar fractures. After 4 years, she suffered right humeral and right index finger fractures. She underwent preventive surgery of the right humerus and left femur 5.5 years after the surgery.

Postoperative X-ray image findings: Kyphosis was corrected from $84^{\circ}$ to $35^{\circ}$ between T3 and L3 (Fig. 4a). Local kyphosis improved from $51^{\circ}$ to $16^{\circ}$ between T11 and L1. Six years after surgery, the kyphosis of T3-L3 remained at $39^{\circ}$ without any progression. No vertebral fractures had occurred at the time of last follow-up (Fig. 4b).

\section{Discussion}

Gaucher's disease makes the patient susceptible to thrombocytopenia, and thus to bleeding, bone fragility, and pathological fractures. It has been reported that if accumulated in the brain, lungs, or bronchi, glucocerebroside may cause convulsions and respiratory dysfunction ${ }^{6}$. For successful surgery, it is essential to assess both the spine and overall physical condition of the patient and coordinate with the pediatrician and the anesthetist.

To the best of our knowledge, 10 cases of vertebral 

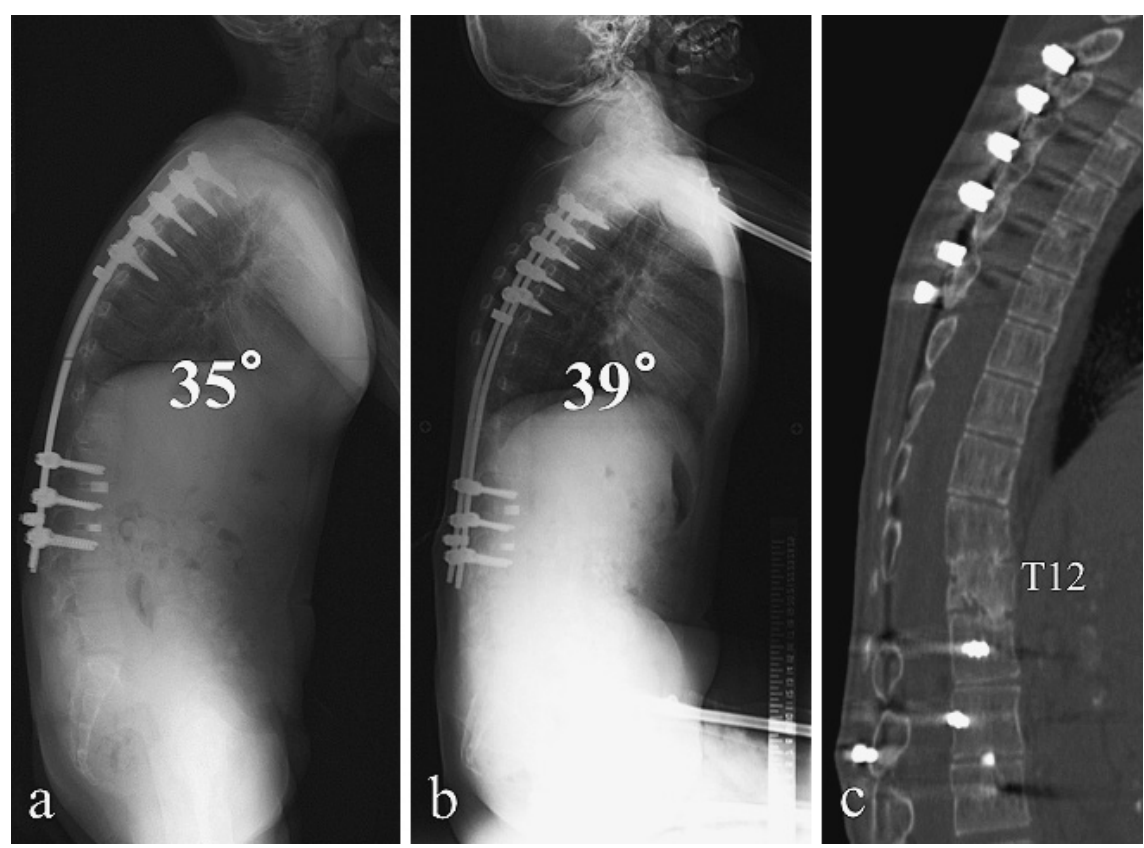

Figure 4. (a) Postoperative radiograph. Kyphosis was corrected from $84^{\circ}$ to $35^{\circ}$ between T6 and L3. Local kyphosis improved from $51^{\circ}$ to $16^{\circ}$. Lumbar lordosis decreased from $26^{\circ}$ to $6^{\circ}$. (b) Final follow-up radiograph ( 6 years after surgery). The kyphosis was maintained at $39^{\circ}$ without vertebral fracture at the latest follow-up. (c) Final follow-up computed tomography (6 years after surgery). T12 pedicle subtraction osteotomy was performed, and the thoracolumbar junction became flat.

kyphosis associated with Gaucher's disease have been reported. Kyphosis was caused by compression fractures in eight of the 10 cases, with only two cases of kyphosis unassociated with compression fractures. In the two cases, reports included no record of surgery and gave no guidance as to the possible causes of kyphosis ${ }^{3-5}$. As for the cause of anterior vertebral wedging, despite the absence of a past history of compression fractures, the condition may be attributable to repeated minor fractures or growth cessation of the end plate, because of glucocerebroside.

A surgical treatment strategy was essential because of vertebral body fragility and anterior vertebral wedging. Hence, we recognized the need for anterior-column reconstruction of the vertebral bodies. We chose to limit the range of fusion between the end vertebrae of the kyphosis to conserve mobile segments in the lumbar spine. This, however, poses a danger of compression lumbar fracture in the vertebrae adjacent to the fusion because of bone fragility. Finally, kyphosis correction was achieved, and the patient has had no particular episode regarding vertebral condition postoperatively. Because of bone fragility, careful selection of the fusion range and careful postoperative therapy was necessary.
Conflicts of Interest: The authors declare that there are no conflicts of interest.

\section{References}

1. Brady RO, Kanfer JN, Shapiro D. METABOLISM OF GLUCOCEREBROSIDES. II. EVIDENCE OF AN ENZYMATIC DEFICIENCY IN GAUCHER'S DISEASE. Biochemical and biophysical research communications. 1965;18:221-5. eng.

2. Meikle PJ, Hopwood JJ, Clague AE, et al. Prevalence of lysosomal storage disorders. Jama. 1999;281(3):249-54. eng.

3. Katz K, Sabato S, Horev G, et al. Spinal involvement in children and adolescents with Gaucher disease. Spine. 1993;18(3):332-5. eng.

4. Kocher MS, Hall JE. Surgical management of spinal involvement in children and adolescents with Gaucher's disease. Journal of pediatric orthopedics. 2000;20(3):383-8. eng.

5. Ruff ME, Weis LD, Kean JR. Acute thoracic kyphosis in Gaucher's disease. A case report. Spine. 1984;9(8):835-7. eng.

6. Cox TM, Schofield JP. Gaucher's disease: clinical features and natural history. Bailliere's clinical haematology. 1997;10(4):65789. eng.

Spine Surgery and Related Research is an Open Access article distributed under the Creative Commons Attribution - NonCommercial - NoDerivatives 4.0 International License. To view the details of this license, please visit (https://creativecommons.org/licenses/by - nc - nd/4.0/). 